\title{
LINEAR DEPENDENCE OF POWERS OF LINEAR FORMS
}

\author{
ANDRZEJ SŁADEK
}

\begin{abstract}
The main goal of the paper is to examine the dimension of the vector space spanned by powers of linear forms. We also find a lower bound for the number of summands in the presentation of zero form as a sum of $d$-th powers of linear forms.
\end{abstract}

\section{Introduction and terminology}

B. Reznick, in his 2003 paper [4 introduced the ticket

$$
T(F)=\left\{d \in \mathbb{N}:\left\{f_{j}^{d}\right\} \text { is linearly dependent }\right\}
$$

for any finite set of polynomials $F=\left\{f_{j}\right\}$. If $f_{j}^{\prime}$ is the homogenisation of the polynomial $f_{j}$, then $T(F)=T\left(F^{\prime}\right)$, where $F^{\prime}=\left\{f_{j}^{\prime}\right\}$. Thus, examination of tickets can be confined to forms. Observing interesting results in Reznick's paper one can ask about "degree" of linear dependence of the set of powers of forms within its ticket, i.e. $\operatorname{dim} \operatorname{span}\left\{f_{j}^{d}\right\}$ for $d \in T(F)$. The question seems to be difficult in the case of any sets of forms. The ticket of a set of linear forms is an initial segment of the set $\mathbb{N}$ of natural numbers (see [4, Lemma 2.2]) and the problem has a chance for at least partial solution in this case.

Received: 6.01.2015. Revised: 12.05.2015.

(2010) Mathematics Subject Classification: 11E76, 15A99.

Key words and phrases: linear form, sums of powers of linear forms, ticket of the set of polynomials. 
In section 2 we examine the growth of the sequence $\left(\operatorname{dim} \operatorname{span}\left\{l_{j}^{d}\right\}\right)_{d \in \mathbb{N}}$ for a finite set $\left\{l_{j}\right\}$ of linear forms. In section 3 we find a lower bound for the number of summands in the presentation of zero form as a sum of $d$-th powers of linear forms. This is connected with a conjecture formulated by A. Schinzel.

Throughout the paper the field $K$ is of characteristic 0 , however some obtained results hold true for fields with big enough (greater than members of considered tickets) positive characteristic. Let $L_{K}(n)$ be the set of linear forms over a field $K$. For any $A=\left\{l_{1}, \ldots, l_{r}\right\} \subset L_{K}(n)$ and $d \in \mathbb{N}$ let $A^{d}$ denote the subset $\left\{l_{1}^{d}, \ldots, l_{r}^{d}\right\}$ of the vector space $F_{K}(n, d)$ of homogeneous forms in $n$ variables of degree $d$ over $K$ and let $w_{d}(A)=\operatorname{dim} \operatorname{span}\left(A^{d}\right)$. We need one more invariant

$$
s_{d}(A)=\max \left\{k: B \subset A, \# B=k \Longrightarrow B^{d} \text { is linearly independent }\right\} .
$$

Of course, by the definition $s_{d}(A) \leq w_{d}(A) \leq \# A$. Throughout the paper we consider distinct elements of $L_{K}(n)$ as projectively distinct, i.e. any $l_{1}, l_{2} \in$ $L_{K}(n), l_{1} \neq l_{2}$, are supposed to be linearly independent. Thus, if $\# A \geq 2$, then $2 \leq s_{1}(A) \leq n$. Let us start with a simple lemma.

Lemma 1.1. Assume $l_{1}, \ldots, l_{r} \in L_{K}(n), \lambda_{1}, \ldots, \lambda_{r} \in K$ and

$$
\sum_{j=1}^{r} \lambda_{j} l_{j}^{d}=0 .
$$

Then for every $\alpha=\left[a_{1}, \ldots, a_{n}\right] \in K^{n}$, we have

$$
\sum_{j=1}^{r} \lambda_{j} l_{j}(\alpha) l_{j}^{d-1}=0 .
$$

Proof. It suffices to apply the differential operator $\frac{1}{d} \frac{\partial}{\partial l}$ to the equation (1.1), where $l=a_{1} X_{1}+\ldots+a_{n} X_{n}$.

Corollary 1.2. For any finite subset $A$ of $L_{K}(n)$ the sequences $\left(s_{d}(A)\right)_{d \in \mathbb{N}}$ and $\left(w_{d}(A)\right)_{d \in \mathbb{N}}$ are nondecreasing.

In the next section we will show that these sequences are increasing until the moment they stabilize. 


\section{Powers of linear forms and the dimensions}

Let us start this section with the main theorem.

Theorem 2.1. Assume $A \subset L_{K}(n), d \geq 2,\left(l_{1}^{d-1}, \ldots, l_{w_{d-1}(A)}^{d-1}\right)$ is a basis of $A^{d-1}$ and $l_{i_{1}}, \ldots, l_{i_{k}} \in A$ satisfy the following conditions:

(a) $\left\{l_{i_{1}}, \ldots, l_{i_{k}}\right\} \cap\left\{l_{1}, \ldots, l_{w_{d-1}(A)}\right\}=\emptyset$,

(b) $l_{i_{1}}, \ldots, l_{i_{k}}$ are linearly independent,

(c) at most $s_{d-1}(A)-1$ of $l_{1}, \ldots, l_{w_{d-1}(A)}$ belong to $\operatorname{span}\left(l_{i_{1}}, \ldots, l_{i_{k}}\right)$.

Then

$$
w_{d}(A)-w_{d-1}(A) \geq k .
$$

PROof. By Corollary 1.2 the sets $\left\{l_{1}^{d}, \ldots, l_{w_{d-1}(A)}^{d}\right\}$ and $\left\{l_{i_{1}}^{d}, \ldots, l_{i_{k}}^{d}\right\}$ are linearly independent. Thus, it suffices to show that

$$
\operatorname{span}\left(l_{i_{1}}^{d}, \ldots, l_{i_{k}}^{d}\right) \cap \operatorname{span}\left(l_{1}^{d}, \ldots, l_{w_{d-1}(A)}^{d}\right)=\{\theta\} .
$$

Suppose

$$
b_{1} l_{i_{1}}^{d}+\ldots+b_{k} l_{i_{k}}^{d}=c_{1} l_{1}^{d}+\ldots+c_{w_{d-1}(A)} l_{w_{d-1}(A)}^{d}, \quad b_{i}, c_{j} \in K, b_{1} \neq 0 .
$$

Let

$$
l_{i_{1}}^{d-1}=\sum_{j=1}^{w_{d-1}(A)} a_{j} l_{j}^{d-1} .
$$

By the definition of $s_{d-1}(A)$, at least $s_{d-1}(A)$ of $a_{1}, \ldots, a_{w_{d-1}(A)}$ must be different from zero. Thus by (c), there exists $j_{0}$ such that $a_{j_{0}} \neq 0$ and the set $\left\{l_{i_{1}}, \ldots, l_{i_{k}}, l_{j_{0}}\right\}$ is linearly independent. We can take $\alpha \in K^{n}$ such that

$$
l_{i_{1}}(\alpha) \neq 0, \quad l_{i_{2}}(\alpha)=\ldots=l_{i_{k}}(\alpha)=l_{j_{0}}(\alpha)=0 .
$$

If $\alpha=\left[a_{1}, \ldots, a_{n}\right]$ and $l=a_{1} X_{1}+\ldots+a_{n} X_{n}$, then after applying differential operator $\frac{1}{d} \frac{\partial}{\partial l}$ to both sides of the equation 2.1 we get

$$
b_{1} l_{i_{1}}(\alpha) l_{i_{1}}^{d-1}=\sum_{i=1, i \neq j_{0}}^{w_{d-1}(A)} c_{i} l_{i}(\alpha) l_{i}^{d-1},
$$

which is in contradiction with 2.2. 


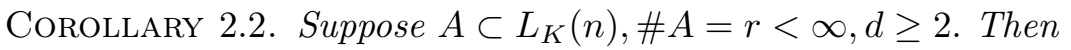

(a) $w_{d}(A)-w_{d-1}(A) \geq \min \left\{s_{1}(A)-1, r-w_{d-1}(A)\right\}$;

(b) if $r-w_{d-1}(A) \geq s_{1}(A)-1$, then $w_{d}(A) \geq(d-1)\left(s_{1}(A)-1\right)+w_{1}(A)$;

(c) if $s_{1}(A)=n$ and $r=d(n-1)+1$, then $A^{d}$ is linearly independent;

(d) the sequence $\left.\left(w_{d}(A)\right)_{d \in \mathbb{N}}\right)$ is increasing untill the $d$ for which $A^{d}$ is linearly independent, $w_{d}(A)=r$ for $d \geq r-1$;

(e) the sequence $\left.\left(s_{d}(A)\right)_{d \in \mathbb{N}}\right)$ is increasing untill the $d$ for which $A^{d}$ is linearly independent, $s_{d}(A)=r$ for $d \geq r-1$;

(f) $s_{d}(A) \geq \min \left\{s_{1}(A)+d-1, r\right\}$.

Proof. (a) Let $k=\min \left\{s_{1}(A)-1, r-w_{d-1}(A)\right\}$ and let $\left(l_{1}^{d-1}, \ldots, l_{w_{d-1}(A)}^{d-1}\right)$ be a basis of $A^{d-1}$. Any linearly independent subset $\left\{l_{i_{1}}, \ldots, l_{i_{k}}\right\}$ of $A$ which is disjoint with $\left\{l_{1}, \ldots, l_{w_{d-1}(A)}\right\}$ satisfies assumptions of Theorem 2.1. Thus, $w_{d}(A)-w_{d-1}(A) \geq k$.

(b) If $r-w_{d-1}(A) \geq s_{1}(A)-1$, then $r-w_{k}(A) \geq s_{1}(A)-1$ for every $k \leq d-1$ and by (a)

$$
w_{d}(A)=\sum_{k=2}^{d}\left(w_{k}(A)-w_{k-1}(A)\right)+w_{1}(A) \geq(d-1)\left(s_{1}(A)-1\right)+w_{1}(A) .
$$

(c) If $s_{1}(A)=n$, then $w_{1}(A)=n$ and the statement results immediately from (b).

(d) It follows easily from (a).

(e) If $r>s_{d}(A)=s_{d-1}(A)$, then there exists $B \subset A, \# B=s_{d}(A)+1$ such that $B^{d}$ is linearly dependent. Take $B_{1} \subset B, \# B_{1}=s_{d}(A)$. Then

$$
\begin{aligned}
s_{d}(A)+1 & >\operatorname{dimspan} B^{d} \geq \operatorname{dim} \operatorname{span} B^{d-1} \\
& \geq \operatorname{dim} \operatorname{span} B_{1}^{d-1}=s_{d-1}(A)=s_{d}(A)
\end{aligned}
$$

and $w_{d}(B)=\operatorname{dim} \operatorname{span} B^{d}=\operatorname{dim} \operatorname{span} B^{d-1}=w_{d-1}(B)$. By (d) the set $B^{d}$ is linearly independent and we obtained a contradiction.

(f) We perform an induction on $d$. For obvious reasons the inequality is also true for $d=1$. Suppose $d \geq 2$ and $s_{d-1}(A) \geq \min \left\{s_{1}(A)+d-2, r\right\}$. If $s_{d}(A) \geq r$, then $s_{d}(A)=r$ and we are done. Suppose $r>s_{d}(A)$ and $s_{1}(A)+d-1>s_{d}(A)$. Since the sequence $\left(s_{d}(A)\right)_{d \in \mathbb{N}}$ is nondecreasing, we have

$$
s_{1}(A)+d-1>s_{d}(A) \geq s_{d-1}(A) \geq s_{1}(A)+d-2 .
$$

Thus, $r>s_{d}(A)=s_{d-1}(A)$ which by (e) is impossible. 
Remark. The statement (c) in Corollary 2.2 was proved by A. BiałynickiBirula and A. Schinzel [1, Theorem 2] for fields of characteristic 0 or greater than $d$. Moreover, they showed an example that the statement is no longer true when $r=d(n-1)+2 \leq \# K+1$.

The lower bound for the difference of two consecutive elements of the sequence $\left(w_{d}(A)\right)_{d \in \mathbb{N}}$ in Theorem 2.1 does not exceed the number of variables. It is not hard to show examples that this bound is strict. However, there are examples of sets of linear forms for which $w_{d}(A)-w_{d-1}(A)$ is much bigger. For example, if

$$
A=\left\{a_{1} X_{1}+\ldots+a_{n} X_{n}: a_{1}, \ldots, a_{n} \in \mathbb{N} \cup\{0\}, \sum_{i=1}^{n} a_{i}=d\right\}
$$

then $A^{d}$ is a basis for $F_{\mathbb{R}}(n, d)$ (see [3, Proposition 2.11]) and

$$
w_{d}(A)-w_{d-1}(A) \geq \operatorname{dim} F_{\mathbb{R}}(n, d)-\operatorname{dim} F_{\mathbb{R}}(n, d-1)=\left(\begin{array}{c}
n+d-2 \\
n-2
\end{array}\right) .
$$

The statement (c) in 2.2 indicates that $w_{d}(A)$ strongly depends on the configuration of the linear forms in $A$, so it is natural to ask what can we say about $w_{d}(A)$ if we have information on linearly independent subsets of $\mathrm{A}$. We conclude this section with citing two interesting results due to A. Chlebowicz and M. Wołowiec-Musiał [2] which shed light on this problem.

Lemma 2.3 ([2, Lemma 2.4]). Let $A=\left\{l_{1}, \ldots, l_{m}\right\} \subset L_{K}(n)$. Suppose there exists a number $k$ and subsets $A_{i 1}, \ldots, A_{i k}$ of $A$ for $i=1, \ldots, m$ such that

(a) $A=\bigcup_{j=1}^{k} A_{i j}, i=1, \ldots, m$,

(b) $l_{i} \notin \operatorname{span}\left(A_{i j} \backslash\left\{l_{i}\right\}\right), i=1, \ldots, m, j=1, \ldots, k$.

Then $A^{k}$ is linearly independent.

The proof of this lemma based on very useful criterion due to P. Serret (see [3, Proposition 2.6]). Actually the original Serret's Theorem refers to the field $\mathbb{R}$, but one can check that its proof holds true for any field of characteristic 0 . The following theorem is an easy consequence of the above lemma.

Theorem 2.4 ([2, Theorem 2.5]). If $A \subset L_{K}(n)$ can be decomposed into a union of $s$ subsets, which are disjoint and linearly independent, then $A^{2 s-1}$ is linearly independent. 
Remark. B. Reznick (see [4, Lemma 3.2]) proved inequality $\# T(A)<$ $\# A-1$ for any finite subset $A$ of $L_{K}(n)$. If $A$ is as in Lemma 2.3, then we have the better bound $\# T(A)<k$. If $A$ is a disjoint union of $s$ linearly independent subsets, then $\# T(A)<2 s-2$.

Lemma 2.3 and Theorem 2.4 turn out to be very useful in constructing examples.

ExAmple. Let $A=\left\{l_{1}, \ldots, l_{n}, l_{1}+l_{n}, \ldots, l_{n-1}+l_{n}\right\} \subset L_{K}(n)$, where $l_{1}, \ldots, l_{n}$ are linearly independent. The sets

$$
A_{i 1}=\left\{l_{1}, \ldots, l_{i-1}, l_{i+1}, \ldots, l_{n}\right\}, \quad A_{i 2}=\left\{l_{1}+l_{n}, \ldots, l_{n-1}+l_{n}, l_{i}\right\}
$$

satisfy conditions (a) and (b) from Lemma 2.3 . Thus, $A^{2}$ is linearly independent and $w_{1}(A)=n, w_{d}(A)=2 n-1$ for $d \geq 2$.

We may generalize this example. Let $B_{0}=\left\{l_{1}, \ldots, l_{n}\right\} \subset L_{K}(n)$, where $l_{1}, \ldots, l_{n}$ are linearly independent, $B_{j}=\left\{l_{j}+l_{i}: j=1, \ldots, n, j \neq i\right\}$ and $A=\bigcup_{j=0}^{n} B_{j}$. The sets $A_{i 0}=B_{0} \backslash\left\{l_{i}\right\}, A_{i j}=B_{j} \cup\left\{l_{i}\right\}$ for $j=1, \ldots, n$, satisfy conditions (a) and (b) from Lemma 2.3. Thus, $A^{n+1}$ is linearly independent and $w_{1}(A)=n, w_{d}(A)=n+n(n-1)$ for $d \geq n+1$.

\section{Schinzel's Conjecture}

In this section we will show one more application of Theorem 2.4. A. Schinzel, during his lecture at 18th Czech and Slovak International Conference on Number Theory (2007, Smolenice, Slovakia), presented the following conjecture.

Conjecture. Let $K$ be a field of characteristic 0 or greater than $d$. If $l_{1}, \ldots, l_{r} \in L_{K}(n), \operatorname{dim} \operatorname{span}\left(l_{1}, \ldots, l_{r}\right)=n$ and $\sum_{i=1}^{r} l_{i}^{d}=0$, but no proper subsum is 0 , then $r \geq d(n-1)+2$.

In [1] it was shown that the conjecture holds true for $n \leq 4$ and any $d$. We shall show that a weaker version of this conjecture holds true.

Lemma 3.1. Let $l_{1}, \ldots, l_{k}, m_{1}, \ldots, m_{s} \in L_{K}(n)$ be pairwise projectively distinct. If

$$
l_{1}^{d}+\ldots+l_{k}^{d}=m_{1}^{d}+\ldots+m_{s}^{d}
$$


$\operatorname{dim} \operatorname{span}\left(l_{1}, \ldots, l_{k}\right)=n$ and the set $\left\{l_{1}^{d-1}, \ldots, l_{k}^{d-1}\right\}$ is linearly independent, then $\operatorname{dim} \operatorname{span}\left(m_{1}, \ldots, m_{s}\right)=n$.

Proof. Suppose that $\operatorname{dim} \operatorname{span}\left(m_{1}, \ldots, m_{s}\right)<n$. Then there exists $\alpha=$ $\left[a_{1}, \ldots, a_{n}\right] \in K^{n}, \alpha \neq \theta$, such that $m_{i}(\alpha)=0$ for $i=1, \ldots, s$. Let us take $l=a_{1} X_{1}+\ldots+a_{n} X_{n}$ and apply the differential operator $\frac{1}{d} \frac{\partial}{\partial l}$ to both sides of equation (3.1). We have

$$
\sum_{j=1}^{k} l_{j}(\alpha) l_{j}^{d-1}=\sum_{i=1}^{s} m_{i}(\alpha) m_{i}^{d-1}=0 .
$$

Since dim $\operatorname{span}\left(l_{1}, \ldots, l_{k}\right)=n$, there exists $j_{0} \in\{1, \ldots, k\}$ such that $l_{j_{0}}(\alpha) \neq$ 0 . We get a contradiction with linear independence of $\left\{l_{1}^{d-1}, \ldots, l_{k}^{d-1}\right\}$.

Theorem 3.2. If $l_{1}, \ldots, l_{r} \in L_{K}(n), \operatorname{dim} \operatorname{span}\left(l_{1}, \ldots, l_{r}\right)=n$ and

$$
\sum_{i=1}^{r} l_{i}^{d}=0,
$$

but no proper subsum is 0 , then $r \geq \frac{n(d+1)}{2}$, if $d$ is odd and $r \geq \frac{n(d+2)}{2}$, if $d$ is even.

Proof. First we show that for any $t \leq \frac{d}{2}+1$ we may find disjoint linearly independent subsets $A_{1}, \ldots A_{t}$ of $\left\{l_{1}, \ldots, l_{r}\right\}$, the number of elements of each is $n$, and such that (after reindexing elements of $\left\{l_{1}, \ldots, l_{r}\right\}$ )

$$
A_{1} \cup \ldots \cup A_{t}=\left\{l_{1}, \ldots, l_{n t}\right\} \subset\left\{l_{1}, \ldots, l_{r}\right\} .
$$

By the assumptions, we may find linearly independent subset $A_{1} \subset\left\{l_{1}, \ldots, l_{r}\right\}$. Take the maximal $t$ for which there exist $A_{1}, \ldots, A_{t}$ satisfying the above requirement. If $t \leq \frac{d}{2}+1$ and $t+1>\frac{d}{2}+1$, then we are done. Thus, suppose $t+1 \leq \frac{d}{2}+1$. Then $2 t-1 \leq d-1$ and by Theorem 2.4 , the set $\left\{l_{1}^{d-1}, \ldots, l_{n t}^{d-1}\right\}$ is linearly independent. Let $\alpha=\sqrt[d]{-1}$. We have

$$
l_{1}^{d}+\ldots+l_{n t}^{d}=\left(\alpha l_{n t+1}\right)^{d}+\ldots+\left(\alpha l_{r}\right)^{d}
$$

and by Lemma 3.1. applied over the field $K(\alpha)$, the set $\left\{l_{n t+1}, \ldots, l_{r}\right\}$ contains at least one more linearly independent subset with $n$ elements. We obtained a contradiction with the choice of $t$.

Now, for even $d$ we may take $t=\frac{d}{2}+1$, and then $r \geq n t=\frac{n(d+2)}{2}$. If $d$ is odd, then $t=\frac{d-1}{2}+1$ is possible and $r \geq n t=\frac{n(d+1)}{2}$. 
REMARK. From the above Theorem it follows that Schinzel's conjecture is true for $d=2$ and any $n$, since then $\frac{n(d+2)}{2}=d(n-1)+2$. Unfortunately, our lower bound for $r$ is the worst the bigger $d$ is. It seems that to prove Schinzel's conjecture one should use other tools. It would be interesting to check this conjecture for $d=3$ and any $n$.

\section{References}

[1] Białynicki-Birula A., Schinzel A., Representations of multivariate polynomials by sums of univariate polynomials in linear forms, Colloq. Math. 112 (2008), 201-233. [Corrigendum. Colloq. Math. 125 (2011), 139.]

[2] Chlebowicz A., Wołowiec-Musiał M., Forms with a unique representation as a sum of powers of linear forms, Tatra Mt. Math. Publ. 32 (2005), 33-39.

[3] Reznick B., Sums of even powers of real linear forms, Memoirs Amer. Math. Soc. 96 (1992), no. 463.

[4] Reznick B., Patterns of dependence among powers of polynomials, DIMACS Ser. Discrete Math. Theoret. Comput. Sci. 60 (2003), 101-121.

Institute of Mathematics

UNIVERSity OF Silesia

BANKOWA 14

40-007 Katowice

POLAND

e-mail: andrzej.sladek@us.edu.pl 